\title{
A time-domain veto for binary inspirals search
}

\author{
G M Guidi ${ }^{1}$ \\ Ligo Visitor, California Institute of Technology, Pasadena, CA 91125, USA \\ E-mail: guidi@fis.uniurb.it
}

Received 2 April 2004

Published 28 September 2004

Online at stacks.iop.org/CQG/21/S1767

doi:10.1088/0264-9381/21/20/019

\begin{abstract}
We describe a test to distinguish between actual gravitational waves from binary inspiral and false noise triggers. The test operates in the time domain, and considers the time evolution of the correlator and its statistical distribution. It should distinguish true versus noisy events with the same signal-to-noise ratio and chi-square frequency distribution. A similar test has been applied to S1 LIGO data.
\end{abstract}

PACS numbers: 95.85.Sz, 04.80.Nn, 07.05.Kf

\section{Introduction}

Several gravitational wave detectors are now operating or are in the final stage of commissioning [1]. One of the main astrophysical sources of gravitational waves these detectors are looking for are binary systems containing neutron stars and/or black holes. The search algorithms for these sources are based on good theoretical knowledge of the gravitational signal and exploit the techniques of matched filters [2].

As the gravitational wave signal is expected to be very low, false events can be found in the data analysis. Due to the characteristics of the binary system signal, these false events are possibly triggered by fast non-stationarities of environmental or instrumental origin, and can be vetoed by checking for coincident transients in the auxiliary data channels [3]. However some of them remain and are produced by unidentified causes.

It is then necessary to develop tests based on the characteristics of the events which can distinguish between actual gravitational waves and false noise triggers. A veto widely applied is the $\chi^{2}$ veto [4] which studies the frequency pattern of the triggers and compares it to the theoretical frequency pattern. It has however been observed that some events pass this veto but are presumably false if considered caused by the gravitational wave signal which we are looking for. This can be argued by looking at the time evolution of the statistics used for the detection, which in the case of these spurious events appears to have high and rapidly varying

1 Permanent address: Istituto di Fisica, Università di Urbino, Via S Chiara 27, 61029 Urbino, Italy. 
values, different from what should be observed for true events $[5,6]$. This is a good reason to develop new vetoes that consider the time instead of the frequency patterns of the events.

Here we present one such veto which is based on the construction of a new statistics similar to the usual filter output and find its distribution. The new statistic is calculated from a modified filter output time series, which involves subtracting the output time series that would be expected based on the signal parameters reported by the search algorithm. This makes it possible to construct a test which analyses the maximum of this statistics in a certain interval of time and fixes a threshold on this maximum which depends on the chosen confidence level of the test. A similar test was developed and applied to the first LIGO science run (S1) data [6].

The paper is organized as follows. Section 2 contains a description of the matched filter techniques and the definition of the new statistics we use to construct our veto. Section 3 describes the veto and its implementation. Section 4 summarizes the results and discusses the properties of the veto.

\section{The matched filter and the correlator statistics}

The gravitational signal produced in the detector can be written as [5]

$$
h(t)=\frac{1 \mathrm{Mpc}}{D_{\text {eff }}}\left[\sin \alpha h_{s}^{I}(t)+\cos \alpha h_{c}^{I}(t)\right],
$$

where $h_{s, c}^{I}(t)$ are the two polarizations produced by an inspiralling binary of masses $I=\left(m_{1}, m_{2}\right)$ that is optimally oriented at a distance of $1 \mathrm{Mpc}$. $h_{c}^{I}$ is the $\alpha=0$ waveform while $h_{s}^{I}$ is the $\alpha=\pi / 2$ waveform, where $\alpha$ depends on the orbital phase and orientation of the binary system. The effective distance $D_{\text {eff }}$ depends on the true distance $r$ to the binary, its location in the sky relative to the detector, and its orientation.

The search for inspiral waveforms is usually done by exploiting the techniques of matched filtering, which consist in evaluating a weighted correlation between the data and a template waveform for all possible coalescence time [2]. The templates we use are second-order postNewtonian waveforms [7] parametrized by the masses of the binaries and by the starting orbital phase, and can be identified with the $h_{c, s}^{I}$. As they depend on the masses of binaries, a bank of templates that covers the expected range of masses must be used.

The two templates $h_{c, s}^{I}$ have a time length $T$ which depends on the masses and on a low-frequency cut-off due to the sensitivity curve of the detector. We can also approximately consider $T$ as the time for the gravitational wave signal to traverse the sensitivity band of the detector. The weighted correlation between the detector's data $s(t)$, and the $\alpha=0, \pi / 2$ templates can be written as

$$
x_{c, s}(t)=2 \int_{-\infty}^{\infty} \frac{\tilde{h}_{c, s}^{I}(f) \tilde{s}^{*}(f)}{S_{n}(f)} \mathrm{e}^{2 \pi \mathrm{i} f t} \mathrm{~d} f,
$$

where $\tilde{h}_{c, s}^{I}(f)$ and $\tilde{s}(f)$ are the Fourier transforms of the templates and of the detector data respectively, and $S_{n}(f)$ is the noise spectral density. The detector data can be written as $s(t)=n(t)+h(t)$, where $n(t)$ is the detector noise, which we suppose to be stationary and Gaussian, and $h(t)$ is the gravitational wave signal of equation (1).

The statistics used for the matched filter is

$$
\rho(t)=\frac{\sqrt{x_{s}^{2}(t)+x_{c}^{2}(t)}}{\sigma} .
$$

In equation (3) $\sigma$ is the variance of the matched filter output due to detector noise [5]. To detect a signal we threshold on the square of this statistics, which is called the signal-to-noise ratio (SNR). 


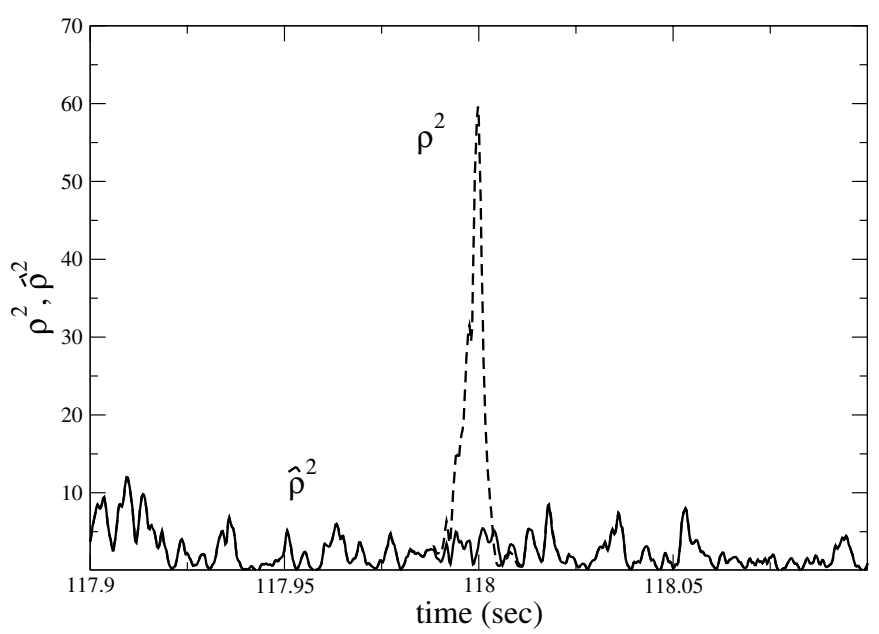

Figure 1. The $\rho^{2}$ and $\hat{\rho}^{2}$ time series for a simulated binary system with masses of 1.4 and $2.0 M_{\odot}$ and $\mathrm{SNR}=8.0$. Here the templates have the same masses as the simulated binary system. The low-frequency cut-off is $30 \mathrm{~Hz}$. The sensitivity used is the target sensitivity of the LIGO $4 \mathrm{~km}$ interferometer design. The two time series are different near the coalescence, where the correlations between the templates and the signal are markedly different from zero. The simulations are done using routines in the LSC Algorithm Library (LAL) [9].

When the signal $h(t)$ is not present $x_{c, s}(t)$ are Gaussian variables with variance $\sigma$ and mean zero. The SNR squared is then $\chi^{2}$ distributed with $v=2$ degrees of freedom.

Suppose now that we detect a signal $h(t)$ at time $t^{(d)}$ by thresholding on $\rho^{2}(t)$ : the SNR

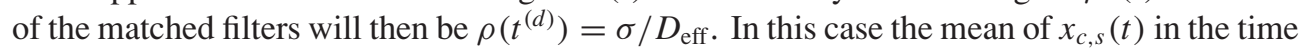
interval $\left[t^{(d)}-T, t^{(d)}+T\right]$ does not vanish but depends on the correlation of the signal with the templates [8], and consequently $\rho^{2}(t)$ is not distributed as a $\chi^{2}$ variable. If we suppose that the gravitational waveform $h(t)$ is exactly described by the templates that match the signal $h_{c, s}^{(d), I}$, this mean can be found as

$$
\begin{aligned}
\left\langle x_{c, s}(\tau)\right\rangle & =2 \int_{-\infty}^{\infty} \frac{\tilde{h}_{c, s}^{(d), I}(f) \tilde{h}^{*}(f)}{S_{n}(f)} \mathrm{e}^{2 \pi \mathrm{i} f(\tau)} \mathrm{d} f \\
& =\frac{1 \mathrm{Mpc}}{D_{\text {eff }}}\left[\sin \alpha\left(h_{c, s}^{(d), I} \mid h_{s}^{(d), I}\right)(\tau)+\cos \alpha\left(h_{c, s}^{(d), I} \mid h_{c}^{(d), I}\right)(\tau)\right],
\end{aligned}
$$

where we have indicated with $(a \mid b)(t)$ the weighted correlations of equation (2) between two time series $a$ and $b$ and have chosen the detection time as time zero ${ }^{2}$. The orbital frequency $\alpha$ and the effective distance $D_{\text {eff }}$ can be evaluated by considering the value of $x_{c, s}(t)$ and the SNR at the time of detection, respectively.

Now we use the mean of equation (4) to construct the new statistics:

$$
\hat{\rho}^{2}(\tau)=\frac{\left(x_{s}(\tau)-\left\langle x_{s}(\tau)\right\rangle\right)^{2}+\left(x_{c}(\tau)-\left\langle x_{c}(\tau)\right\rangle\right)^{2}}{\sigma^{2}},
$$

which has a $\chi^{2}$ distribution even in the presence of the gravitational wave signal that we suppose has been detected. The subtraction of $\left\langle x_{c, s}(\tau)\right\rangle$ reduces the $\hat{\rho}^{2}(\tau)$ in the $\rho^{2}(\tau)$ of equation (3) that would have been observed in the same time interval if the gravitational wave signal did not arrive (see figure 1). Thus $\hat{\rho}^{2}(\tau)$ depends only on the detector noise that

2 Note that the correlation between the two polarizations is symmetric with respect to the detection time, i.e. $\left(h_{c}^{(d), I} \mid h_{s}^{(d), I}\right)(\tau)=\left(h_{s}^{(d), I} \mid h_{c}^{(d), I}\right)(-\tau)$; this symmetry can be exploited to compute the mean. 
we want to analyse: we can study the time evolution of $\hat{\rho}^{2}(\tau)$ around the detection time to see if it has the same characteristics as $\rho^{2}(\tau)$ in the absence of gravitational wave signals. It is evident from figure 1 that $\rho^{2}(\tau)$ and $\hat{\rho}^{2}(\tau)$ differ only near the detection time, as the correlation between the templates and the signal dies off quickly in a correlation time that can be considered proportional to the inverse of the low-frequency cut-off.

The occurrence that the gravitational waveform is exactly described by the templates obviously represents an ideal situation: usually the templates that match the signal do not have the same masses as the signal as they are chosen from a discrete bank of templates that is constructed in such a way to cover the range of expected masses. In this case, the statistic $\hat{\rho}^{2}(\tau)$ does not exactly reduce to $\rho^{2}(\tau)$ and thus it cannot be considered fully independent of the gravitational wave signal. It is interesting to have an indication of the strength of this effect to see if it is still possible to safely apply the veto that will be described in the following section.

For this purpose, we constructed a bank designed to cause less than $3 \%$ loss in the SNR due to parameter mismatches between any waveform and the nearest template in the bank. We then simulated three signals with masses of the two companions of $I=[1.4,1.4],[1.4,2.0]$ and $[1.4,3.0]$ solar masses and for each of them we chose in the bank the templates with a pair of masses $I^{\prime}$ that causes a loss in the SNR near 3\%. The masses of the templates were: $I^{\prime}=[1.1126,1.7789],[1.3305,2.1135]$ and [1.3027, 3.2552] solar masses respectively. $\hat{\rho}^{2}$ was then evaluated by computing the expressions of equation (4) using the templates of masses $I^{\prime}$, as would have been done in the detection of signals from binary systems with unknown masses.

The $\rho^{2}$ and $\hat{\rho}^{2}$ time series for the detection of these three simulated binary systems are shown in figure 2 . The subtraction of the effect due to the incoming wave seems to be effective also in these cases, even if the $\hat{\rho}^{2}$ time series cannot be exactly the same as obtained with an ideal template.

\section{The veto}

As can be seen in figure 1, the SNR time series for a true signal rises very sharply at the detection time, whereas it is low around it. However it has been observed that false noise triggers often show irregular behaviour and high values before the detection time [5]. This behaviour could be linked to a fast non-stationarity in the noise that triggers the detection. Most of those events are vetoed with a $\chi^{2}$ analysis of the contribution to the SNR from a number of frequency sub-bands as compared to the expected contribution for the templates [4], but some irregular events can pass this test [5]. To veto an event with this characteristic we can construct a test which analyses the SNR time series before the coalescence. A test of this kind has been used to analyse data from $\mathrm{S} 1$ and it has been found effective to eliminate supposed false noise triggers that passed the $\chi^{2}$ test [6].

We consider as statistical variable for our veto the maximum of the $\hat{\rho}^{2}$ time series in a time interval $\Delta t$ before the detection time. We thus have to find the statistical distribution of the maximum extracted from a set of $N$ data, where in our case $N$ is the number of time samples of $\hat{\rho}^{2}$ in $\Delta t$. If the data are independent and identically distributed (iid), one may easily compute the cumulative distribution of maxima as the product of the cumulative distributions. Here we cannot use this approach because the $\hat{\rho}^{2}$ samples are not independent as $x_{c, s}(t)$ have a nonzero correlation time [8]. We have to use an extreme value distribution which describes the maximum of a set of random variables, the Fréchet distribution, which is described in the appendix [10]. 


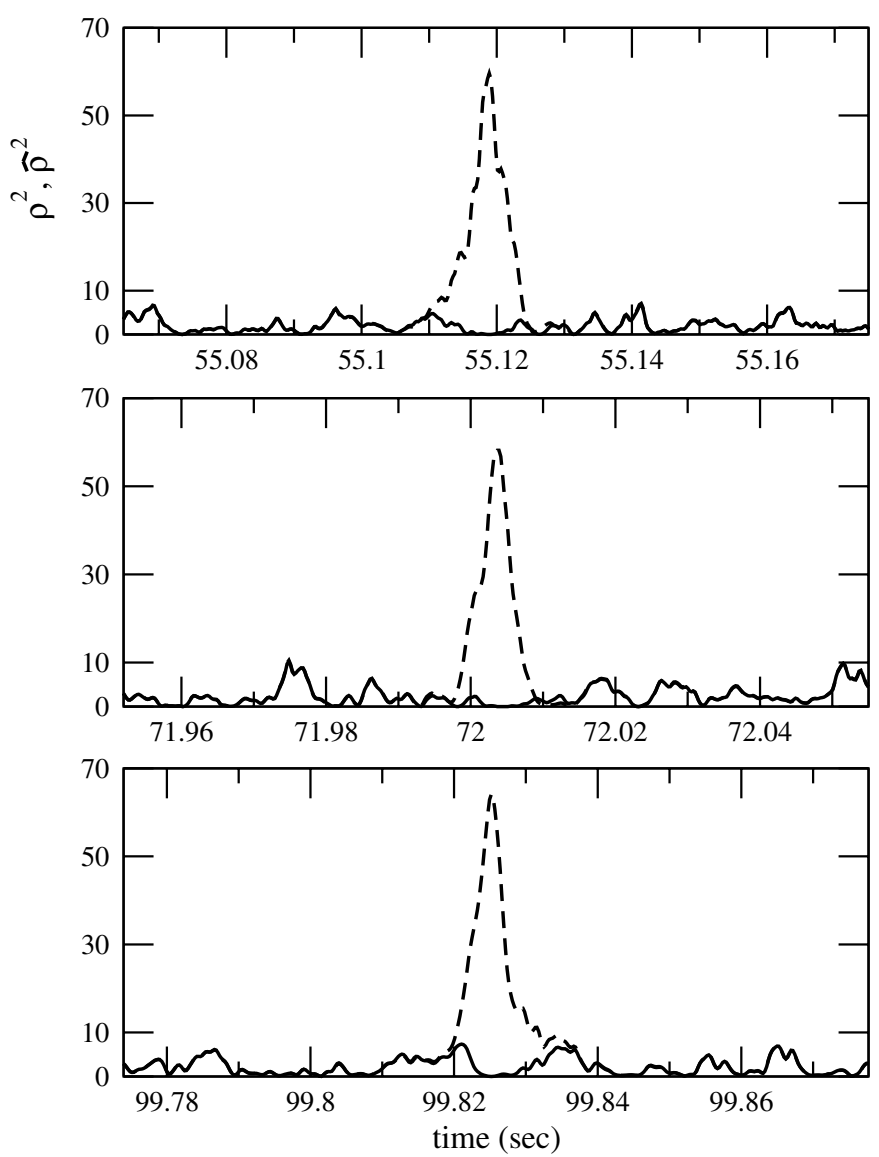

Figure 2. The $\rho^{2}$ (dashed line) and $\hat{\rho}^{2}$ (plain line) time series for simulated binary systems with SNR $=8.0$. The masses of the simulated signals and of the templates are different: $[1.4,1.4] M_{\odot}$ and $[1.1126,1.7789] M_{\odot}$ respectively in the upper panel; $[1.4,2.0] M_{\odot}$ and $[1.3305,2.1135] M_{\odot}$ in the middle panel; $[1.4,3.0] M_{\odot}$ and $[1.3027,3.2552] M_{\odot}$ in the bottom panel.

The Fréchet $\hat{\rho}_{\max }^{2}$ distribution is shown in figure 3. This distribution is obtained by dividing into subsequent non-overlapping intervals of length $\Delta t$ a playground data set which is representative of the main data set and finding the $\hat{\rho}_{\max }^{2}$ for each interval. As the values of $\hat{\rho}^{2}$ depend on the templates that match the signal, this distribution has to be estimated for each different event we want to analyse.

We can now find the probability that the maximum of $\hat{\rho}^{2}$ in the time interval $\Delta t$ before the coalescence exceeds a threshold $\mathrm{THR}_{\hat{\rho}_{\max }^{2}}$ using the Fréchet cumulative distribution $F_{\gamma, \sigma_{F}}$ from equation (A.2)

$$
P\left(\hat{\rho}_{\max }^{2}>\mathrm{THR}_{\hat{\rho}_{\max }^{2}}\right)=1-F_{\gamma, \sigma_{F}}\left(\mathrm{THR}_{\hat{\rho}_{\max }^{2}}\right) .
$$

Inverting this relation we evaluate the threshold $\mathrm{THR}_{\hat{\rho}_{\max }^{2}}$ corresponding to the probability $C$, that is the confidence level of our test, that $\hat{\rho}_{\max }^{2}$ exceeds this threshold for the actual Gaussian and stationary noise:

$$
\mathrm{THR}_{\hat{\rho}_{\max }^{2}}=\sigma_{F}\left(\ln \frac{1}{1-C}\right)^{-\frac{1}{\gamma}} .
$$




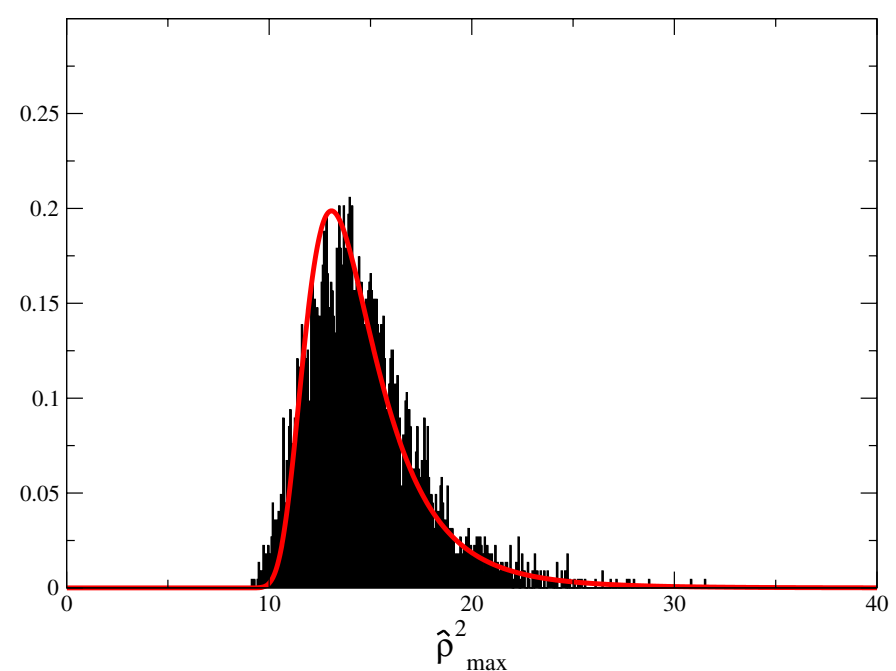

Figure 3. The $\hat{\rho}_{\max }^{2}$ distribution and its approximation by a Fréchet distribution with parameters $\gamma=7.13$ and $\sigma_{F}=1.33$. The time interval for the extraction of the maximum is $\Delta t=1 \mathrm{~s}$. The template parameters are the same as in figure 1.

(This figure is in colour only in the electronic version)

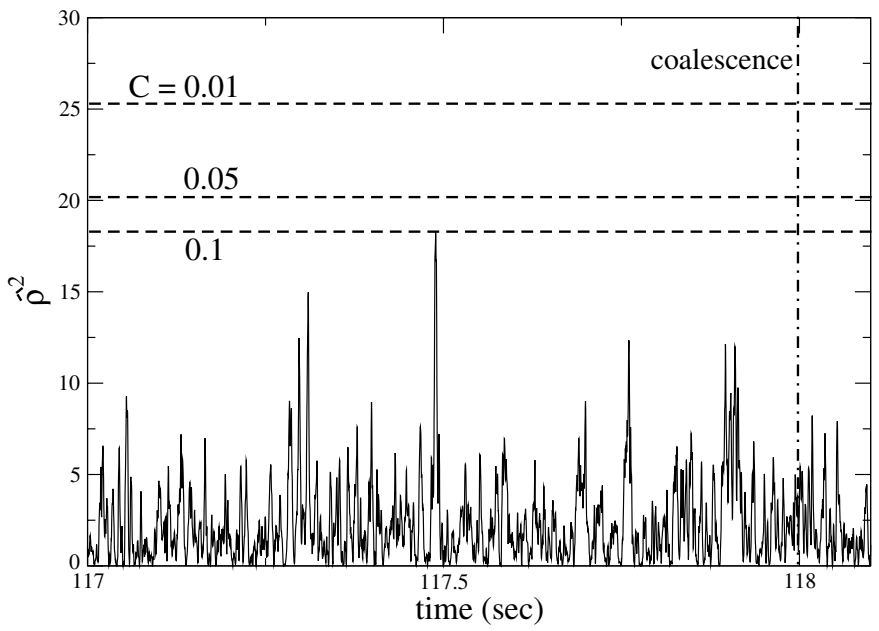

Figure 4. The $\hat{\rho}^{2}$ time series in an interval of $1 \mathrm{~s}$ before the coalescence. Also shown with dashed lines are the thresholds for the maximum for different confidence levels. The maximum has a value of 18.24 which is inferior even to the lower threshold of 18.27 obtained for $C=0.1$.

We can summarize the steps to construct the veto on the maximum for an event:

(1) Choose $\Delta t$ and the confidence level $C$ of the test.

(2) Evaluate the Fréchet parameters $\gamma$ and $\sigma_{F}$ by computing the values of the maximum of $\hat{\rho}^{2}$ in the time intervals $\Delta t$ in the playground data set. Then $\hat{\rho}^{2}$ is computed using the templates that have matched the event. This requires also the estimation of the orbital phase $\alpha$ and of the SNR of the binary system.

(3) Compute the maximum of $\hat{\rho}^{2}$ in the time interval $\Delta t$ before the detection time. 
(4) Compare this maximum with the threshold of equation (7) to decide whether to pass or veto the event at the confidence level chosen.

As an example of different confidence levels $C$ and relative thresholds we report in figure 4 the $\hat{\rho}^{2}$ time series in an interval $\Delta t=1 \mathrm{~s}$ before the coalescence of a binary system with masses of 1.4 and $2.0 M_{\odot}$ and SNR $=8.0$ - the same as figure 1 . This event would have passed the test even at a low confidence level $C=0.1$.

\section{Discussion and conclusions}

We have presented a test for the veto of false noise triggers in the search for gravitational waves from binary inspirals. The test is based on the analysis of a modified-SNR time series before the time of detection. The idea was first exploited by Shawhan and Ochsner in the analysis of the S1 data [6] and is justified by the observation that false noise triggers, in contrast to simulated real detections, often display a high and irregular behaviour before the coalescence. This is evident for example for the largest candidate event seen during the $\mathrm{S} 1$ run that has passed the $\chi^{2}$ frequency veto but has an SNR time series around the detection very different from the simulated injection of a coalescence with the same characteristics [5].

In the work of Shawhan and Ochsner the idea is to consider the number of times the SNR crosses a threshold during a short time before coalescence. Its value for a certain confidence level is found with a Monte Carlo simulation. As the SNR shows the correlation between the signal and the templates near coalescence, the threshold can possibly vary as a function of the SNR of the event.

The statistics $\hat{\rho}^{2}(t)$ of equation (5), which is a modified SNR, allows one to avoid this problem because we subtract from the SNR time series the correlation between the signal and the templates. Moreover, all the $\hat{\rho}^{2}(t)$ are $\chi^{2}$ distributed with $v=2$ degrees of freedom if the detector noise is Gaussian and stationary.

In reality, a discrete template bank is used for the detection and the templates which match the signal normally do not have the same masses as the signal itself. Even if the bank is normally designed as to have a minimal loss in the SNR due to parameter mismatches, this might create some problems as we could not subtract from the SNR time series the exact contribution from the signal. The question surely needs a more extensive analysis. Nevertheless, according to the tests we did (see figure 2), it seems not to be an important effect for large minimal match grids.

We propose to consider for the veto the $\hat{\rho}^{2}(t)$ maximum in a time interval before the coalescence. The time interval depends on the type of non-stationarities which can mimic a coalescence that we expect to find in the data, and has to be chosen by inspection of the data characteristics. The maxima of a iid random variable have a Fréchet distribution which can be estimated from a playground data set. This fact makes it possible to choose the confidence level for the veto of an event and then find the corresponding threshold. This veto can be considered complementary to the standard $\chi^{2}$ test as it considers the filter output over a time interval before the coalescence.

The difference between $\rho^{2}(t)$ and $\hat{\rho}^{2}(t)$ is significant mainly near the coalescence time, thus it can also be possible to find the maximum of $\rho^{2}(t)$ instead of $\hat{\rho}^{2}(t)$ if we previously neglect a little interval of time before the coalescence that could be related to the $\rho^{2}(t)$ time correlation. This would slightly simplify the construction of the veto and needs the estimation of the $\rho^{2}(t)$ time correlation.

It is worth stressing that an event with an irregular behaviour which is rejected by the veto could be due to not well-understood causes, for instance a gravitational wave coupling 
with some nonlinear response of the detector. In this view the veto will be further studied by applying it to real detector data with and without hardware injections simulating different types and strengths of gravitational waves.

\section{Acknowledgments}

I am grateful to Albert Lazzarini and Flavio Vetrano for having made possible my stay at the California Institute of Technology, and to Giancarlo Cella and Peter Shawhan for fruitful discussions. The author has benefited of the LIGO Laboratory Visitors Program with NSF Grant No PHY-0245117.

\section{Appendix. Extreme value distributions}

The distribution of the maximum extracted from a set of $N$ data is the Fréchet distribution [10]:

$$
f_{\gamma, \sigma_{F}}(x)=\frac{1}{\sigma_{F}} \gamma \mathrm{e}^{-\left(\frac{x}{\sigma_{F}}\right)^{-\gamma}}\left(\frac{x}{\sigma_{F}}\right)^{-(1+\gamma)},
$$

where $\gamma$ and $\sigma_{F}$ are called the shape and scale parameters. The cumulative Fréchet distribution is

$$
F_{\gamma, \sigma_{F}}(x)=\mathrm{e}^{-\left(\frac{x}{\sigma_{F}}\right)^{-\gamma}} .
$$

The shape and scale parameters are related to two other parameters, $\mu_{G}$ and $\sigma_{G}$, which enter another extreme value distribution, the Gumbel distribution, which is obtained from the Fréchet distribution through a logarithmic transformation: if $x$ is a Fréchet random variable with shape and scale parameters $\gamma$ and $\sigma_{F}$, then $\ln x$ is a Gumbel random variable with $\ln \sigma_{F}$ and $1 / \gamma$ parameters. The reason to consider the Gumbel distribution of the logarithm of the maximum is that $\mu_{G}$ and $\sigma_{G}$ can be estimated from the sample mean $\overline{\ln x}$ and variance $s_{n}$ of the $\ln x$ :

$$
\sigma_{G}=\frac{\sqrt{6} s_{n}}{\pi}, \quad \mu_{G}=\overline{\ln x}-\sigma_{G} \lambda,
$$

where $\lambda$ is Euler's constant. Thus to find the parameters of the Fréchet distribution we can find the sample mean and variance of the logarithm of the maxima and use the relations:

$$
\gamma=\frac{\pi}{\sqrt{6} s_{n}}, \quad \sigma_{F}=\mathrm{e}^{\overline{\ln x}-\sigma_{G} \lambda}
$$

\section{References}

[1] Here is a list of Internet sites where more information can be found on the main gravitational wave detectors: LIGO (http://www.ligo.caltech.edu), TAMA300 (http://tamago.mtk.nao.ac.jp), GEO600 (http:// www.geo600.uni-hannover.de), VIRGO (http://www.virgo.infn.it), IGEC (http://igec.lnl.infn.it)

[2] McDonough R N and Whalen A D 1995 Detection of Signals in Noise (New York: Academic)

[3] Christensen N et al (LIGO Scientific Collaboration) 2004 Preprint gr-qc/0403114

[4] Allen B et al 1999 Phys. Rev. Lett. 831498

Allen B 2004 Preprint gr-qc/0405045

[5] Abbott B et al (LIGO Scientific Collaboration) 2003 Preprint gr-qc/0308069

[6] Shawahn P and Ochsner E 2004 Class. Quantum Grav. 21 S1757

[7] Blanchet L et al 1996 Class. Quantum Grav. 13575

[8] Dhurandhar S V and Sathyaprakash B S 1994 Phys. Rev. D 491707

[9] LSC Algorithm Library software packages (LAL), Online at http://www.lsc-group.phys.uwm.edu/lal

[10] Reiss R D and Thomas M 2001 Statistical Analysis of Extreme Values (Basle: Birkhäuser) 